\title{
Control of Response Selection by Reinforcer Value Requires Interaction of Amygdala and Orbital Prefrontal Cortex
}

\author{
Mark G. Baxter, ${ }^{1}$ Amanda Parker, ${ }^{2}$ Caroline C. C. Lindner, ${ }^{3}$ Alicia D. Izquierdo, ${ }^{3,4}$ and Elisabeth A. Murray ${ }^{3}$ \\ ${ }^{1}$ Department of Psychology, Harvard University, Cambridge, Massachusetts 02138, 2School of Psychology, University of \\ Nottingham, Nottingham NG7 2RD, United Kingdom, ${ }^{2}$ Laboratory of Neuropsychology, National Institute of Mental \\ Health, Bethesda, Maryland 20892, and ${ }^{4}$ Department of Psychology, The George Washington University, Washington, \\ DC 20052
}

\begin{abstract}
Goal-directed actions are guided by expected outcomes of those actions. Humans with bilateral damage to ventromedial prefrontal cortex, or the amygdala, are deficient in their ability to use information about positive and negative outcomes to guide their choice behavior. Similarly, rats and monkeys with orbital prefrontal or amygdala damage have been found to be impaired in their responses to changing values of outcomes. In the present study, we tested whether direct, functional interaction between the amygdala and the orbital prefrontal cortex is necessary for guiding behavior based on expected outcomes. Unlike control monkeys, rhesus monkeys with surgical disconnection of these two structures, achieved by crossed unilateral
\end{abstract}

lesions of the amygdala in one hemisphere and orbital prefrontal cortex in the other, combined with forebrain commissurotomy, were unable to adjust their choice behavior after a change in the outcome (here, a reduction in the value of a particular reinforcer). The lesions did not affect motivation to work for a food reinforcer, or food preferences, per se. Hence, the amygdala and orbital prefrontal cortex act as part of an integrated neural system guiding decision-making and adaptive response selection.

Key words: amygdala; crossed-lesion technique; decisionmaking; orbital prefrontal cortex; reinforcer devaluation; representation; rhesus monkey
Converging evidence implicates the amygdala and orbital prefrontal cortex, two regions with rich anatomical interconnections (Porrino et al., 1981; Amaral et al., 1992; Carmichael and Price, 1995), in the control of decision-making based on expected outcomes of actions. Humans with damage to the ventromedial prefrontal cortex, including the orbital cortex, or the amygdala, are impaired in using information about positive and negative outcomes to guide their behavioral responses (Bechara et al., 1998, 1999). In addition, monkeys with bilateral excitotoxic lesions of the amygdala fail to alter their actions in response to changes in the value of a reinforcer (Málková et al., 1997); similar results have been reported in rats with neurotoxic lesions of the basolateral amygdala or orbitofrontal cortex (Hatfield et al., 1996; Gallagher et al., 1999). Finally, neurons in both the amygdala and orbital prefrontal cortex signal the value of specific reinforcers and, in addition, the value of the expected outcomes of actions (Rolls, 1996, 1999; Schoenbaum et al., 1998, 1999; Rogers et al., 1999b; Tremblay and Schultz, 1999).

Neither examination of the effects of bilaterally symmetrical lesions nor traditional electrophysiological approaches can indicate whether discrete neural structures interact with one another

\footnotetext{
Received Feb. 10, 2000; revised March 17, 2000; accepted March 17, 2000.

This work was supported by the Intramural Research Program of the National Institute of Mental Health. We thank Richard Saunders for invaluable assistance in surgery, Alison Schaefer for help with behavioral testing, Tim Bussey for programming the progressive ratio task, and Peter Holland and Steve Wise for helpful comments on an earlier version of this manuscript.

Correspondence should be addressed to either Dr. Mark G. Baxter, Department of Psychology, Harvard University, 906 William James Hall, 33 Kirkland Street, Cambridge, MA 02138, E-mail: mbaxter@wjh.harvard.edu, or Dr. Elisabeth A. Murray, Laboratory of Neuropsychology, National Institute of Mental Health, Building 49, Room 1B80, 49 Convent Drive, Bethesda, MD 20892-4415, E-mail: eam@ln.nimh.nih.gov. Reprint requests should be addressed to Elisabeth A. Murray. Copyright (C) 2000 Society for Neuroscience $0270-6474 / 00 / 204311-09 \$ 15.00 / 0$
}

to guide behavior. However, the use of a procedure producing a selective disconnection of two brain regions can reveal essential interactions between neural structures (Ettlinger, 1959). To test whether the amygdala and orbital prefrontal cortex interact in mediating control of response selection based on the relative values of outcomes, we studied the effect of surgical disconnection of these structures in rhesus monkeys (Macaca mulatta) on performance of a reinforcer devaluation task (Málková et al., 1997). In this task, monkeys learn a set of object discrimination problems in which the rewarded objects are paired with one of two distinct reinforcers (foods). They are then confronted with critical test sessions consisting of choices between pairs of positive objects, each of which had been associated with a distinct food during preliminary training. Before some of these sessions, the monkeys were allowed to eat as much as they wanted of one of the two foods, thereby reducing the value of that reinforcer via selective satiation. These two critical sessions challenge the monkeys to make adaptive responses to compensate for the altered value of the outcome. Other critical test sessions, not preceded by a satiation procedure, provide a baseline of choice behavior for each monkey. In practice, when one of the two reinforcers is devalued by selective satiation, normal monkeys tend to avoid choosing objects that are paired with the devalued (satiated) reinforcer compared with their own choice behavior in baseline sessions (Málková et al., 1997; Thornton et al., 1998).

In the present study, monkeys sustained a neurotoxic lesion of the amygdala in one hemisphere and an aspiration lesion of the orbital prefrontal cortex in the opposite hemisphere, combined with forebrain commissurotomy, thereby eliminating interaction between these structures. If such direct interaction between these structures is required to produce adaptive response selection, i.e., a change in choice behavior in response to a change in reinforcer 


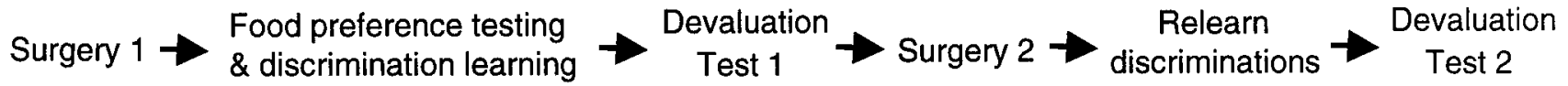

Figure 1. Schematic of testing sequence for the reinforcer devaluation study. Control subjects received no surgery and were given a period of rest equivalent to that provided for the operated monkeys. Note that surgery 2 completes the disconnection of amygdala and orbital prefrontal cortex in all operated subjects.

value, this disconnection surgery should disrupt the reinforcer devaluation effect.

Parts of this work have been published previously in abstract form (Parker et al., 1999).

\section{MATERIALS AND METHODS}

\section{Subjects and experimental design}

Eight experimentally naive rhesus monkeys (Macaca mulatta), all male, were used. They weighed $4.4-6.5 \mathrm{~kg}$ at the beginning of the study, were housed individually in rooms with automatically regulated lighting $(12 \mathrm{hr}$ light/dark, lights on at 7:00 A.M.), and were maintained on primate chow (\#5038, PMI Feeds Inc, St. Louis, MO) supplemented with fresh fruit and peanuts. Water was always available in the home cage.

A schematic of the experimental design is presented in Figure 1. Four monkeys were assigned to the surgical disconnection group (designated cases Op1-Op4), and four additional monkeys were retained as unoperated controls (designated cases Con1-Con4). Within the disconnection group, two monkeys received unilateral ibotenic acid lesions of the amygdala before the beginning of behavioral testing, and the two others received unilateral aspiration lesions of the orbital prefrontal cortex, as well as section of the anterior commissure and corpus callosum, before the beginning of behavioral testing. After the first phase of training had been completed (including accommodation, pretraining, food preference testing, discrimination learning, and reinforcer devaluation test 1), each of the four operated monkeys received the complementary surgery in the opposite hemisphere (unilateral orbital prefrontal ablation and commissurotomy for the monkeys with unilateral amygdala lesions and vice versa). As indicated above, the two surgeries together were designed to prevent the direct functional interaction of the amygdala and orbital prefrontal cortex, while at the same time leaving one of each structure to interact with other brain regions. After the second surgery (or an equivalent period of rest for the controls), all monkeys were reassessed on the reinforcer devaluation task (test 2). The expectation was that the groups would not differ on test 1 , indicating that the unilateral lesions alone were without effect. However, if the amygdala and orbital prefrontal cortex were required to interact to guide monkeys' choice behavior, as we predicted, then the groups would differ on test 2, after the disconnection was complete.

\section{Surgery}

The lesions at each stage were counterbalanced for left-right hemispheres across monkeys; that is, of the two monkeys that received amygdala lesions first, one received a left amygdala lesion and the other received a right amygdala lesion, etc. At the time of surgery, anesthesia was induced with ketamine hydrochloride $(10 \mathrm{mg} / \mathrm{kg}$, i.m.) and maintained with isoflurane $(1.0-2.0 \%$, to effect). The animals received isotonic fluids via an intravenous drip. Aseptic procedures were used. Heart rate, respiration rate, blood pressure, expired $\mathrm{CO}_{2}$, and body temperature were monitored throughout the procedure. For the amygdala lesions, as described in previous papers (Murray et al., 1996; Málková et al., 1997), injections of ibotenic acid were placed stereotaxically throughout the amygdala in one hemisphere, with coordinates determined from magnetic resonance imaging (MRI) scans performed immediately before each surgery. Approximately 21 injections of 0.4 (Op1), 0.6 (Op2 and Op4), or 1.0 (Op3) $\mu \mathrm{l}$ of ibotenic acid $(10 \mathrm{mg} / \mathrm{ml}$; Biosearch Technologies, Novato, CA) were made in the amygdala via the 30 gauge needle of a Hamilton syringe. Each injection was made at the rate of $0.2 \mu \mathrm{l} / \mathrm{min}$, and the needle was left in place $2-3 \mathrm{~min}$ after each injection to limit diffusion of the toxin up the needle track. After the injections were completed, the scalp was closed in anatomical layers. All monkeys received a preoperative and postoperative treatment regimen consisting of dexamethasone sodium phosphate $(0.4 \mathrm{mg} / \mathrm{kg})$ and Cefazolin antibiotic $(15 \mathrm{mg} / \mathrm{kg})$ for $1 \mathrm{~d}$ before surgery and 1 week after surgery to reduce swelling and to prevent infection, respectively. They also received Banamine (flunixin meglumine, $5 \mathrm{mg}$ ) for $3 \mathrm{~d}$ after surgery as an analgesic.
For the orbital prefrontal cortex lesion and commissurotomy, general surgical procedures were identical to those used for the amygdala lesion, except that MRI scans were not required and a regular (not stereotaxic) head holder was used. The anterior commissure and corpus callosum were visualized and sectioned using gentle suction through a fine glass sucker. Then, using a combination of suction and electrocautery, the orbital prefrontal cortex was removed by subpial aspiration through a fine-gauge metal sucker, insulated except at the tip. The intended lesion extended from the fundus of the lateral orbital sulcus laterally to the fundus of the rostral sulcus medially. The rostral limit of the lesion was a line joining the anterior tips of the lateral and medial orbital sulci, and the caudal limit of the lesion was approximately at the anteroposterior level of the rostral extent of the inferior limb of the arcuate sulcus. This lesion was intended to remove the main targets of the amygdala projection to the prefrontal cortex. Although regions of the prefrontal cortex outside our lesion (e.g., anterior cingulate cortex) have been reported to receive a direct projection from the amygdala, the prefrontal cortical fields we removed appear to receive the bulk of the amygdalar projections (Porrino et al., 1981; Amaral and Price, 1984; Carmichael and Price, 1995).

\section{Apparatus and materials}

Monkeys were trained in a manual test apparatus, the Wisconsin General Test Apparatus (WGTA), located in a darkened room. The test compartment was lit with two $60 \mathrm{~W}$ incandescent bulbs, but the monkey's compartment was always unlit. Extraneous sound masking was provided with a white noise generator. The test tray, which was located at the level of the floor of the monkey's transport cage, contained two food wells spaced $290 \mathrm{~mm}$ apart, center to center, on the midline of the tray. The wells were $38 \mathrm{~mm}$ in diameter and $6 \mathrm{~mm}$ deep. The stimuli for discrimination learning were 120 "junk" objects that varied widely in color, shape, and size; three additional objects were used during accommodation and pretraining and were dedicated to these stages. Several gray matboard plaques, measuring $76 \mathrm{~mm}$ on each side, were also used during accommodation. Food rewards consisted of a variety of items: a single banana-flavored pellet (P. J. Noyes Inc., Lancaster, NH), a half-peanut, a raisin, a sweetened dried cranberry (Craisins; Ocean Spray, LakevilleMiddleboro, MA), a "fruit snack" chewy candy made from fruit juice (Giant Foods), or a sugar-coated chocolate candy (M\&Ms).

\section{Behavioral testing procedures}

Accommodation. Monkeys were introduced to the WGTA by allowing them to take food from the test tray. Then they were trained, through successive approximation, to displace plaques completely covering a food well to obtain the food reward hidden underneath. Finally, the monkeys were required to displace objects covering the wells to obtain the food reward underneath.

Food preference testing. The preferences of each monkey for the six foods listed above were determined. Each session consisted of 30 trials with a $30 \mathrm{sec}$ intertrial interval. The trials consisted of pairings of each food item with every other food item. On each trial, one food was placed in each well of the test tray. Each of the 15 possible individual pairings appeared twice in each session, with the left-right positions of the two foods reversed between the two appearances. Hence, each food was encountered 10 times in each session. The trials were presented in a random order each day, and the monkeys were tested for a total of $10 \mathrm{~d}$.

On each trial, the screen was raised to allow the monkey access to the test tray, and the monkey was allowed to choose one food reward. The door was lowered if the monkey attempted to take both foods. If the monkey managed to obtain both foods, the one that it chose first was recorded as the response for that trial.

The data for each monkey were tabulated in terms of total number of choices of each food across the last $5 \mathrm{~d}$ of testing. These data were used to generate an individual ranking of preferences for each food for each monkey. Two foods were selected for each monkey that were approximately equally preferred; one was designated as food 1 and the other as food 2 . 
Pretraining. For one session, after the completion of food preference testing and before the onset of formal discrimination learning, the monkeys were presented with 50 trials with a $20 \mathrm{sec}$ intertrial interval in which a single object (one of the three training objects) covered either the left or right well according to a pseudorandom schedule. On half of these trials, the object was not rewarded; on the other half of these trials, the object covered a food item, either food 1 or food 2, chosen at random. An additional 10 trials were then given in this session in which both wells were covered (with two of the three available objects). On five of these trials, both objects were baited with food rewards (either food 1 or food 2), so regardless of the monkey's choice, it would obtain a reward; on the remaining five trials, both objects were unbaited. This procedure was designed to ensure that the monkey would work consistently for food rewards throughout an entire 60 trial session and to accustom the monkey to seeing two objects on the test tray.

Visual discrimination learning. Monkeys were trained to discriminate 60 pairs of objects. For each pair, one object was arbitrarily designated positive (i.e., baited with a food reward) and the other negative (i.e., not baited). On each trial, the two objects comprising a pair, one positive and one negative, were presented for choice, each overlying one of the two food wells on the test tray. Each pair of objects appeared in only one trial per session, yielding a total of 60 trials per day. Half of the positive objects were randomly assigned to be baited with food 1 and the other half with food 2 . The positive and negative objects within each pair, the food reward assignment, and the order of the pairs remained constant across sessions, but the left-right position of the positive objects in each pair followed a pseudorandom order. The intertrial interval was $20 \mathrm{sec}$. Criterion was set at a mean of $90 \%$ correct responses over 5 consecutive days (i.e., 270 correct responses of 300 ).

Reinforcer devaluation. After the monkeys had attained criterion, their choices of objects were assessed in four critical test sessions (test 1), each performed on a separate day. In these sessions, only the positive objects were used; the negative objects were set aside. Thirty pairs, each consisting of one food 1 and one food 2 object, were randomly generated before each critical test session. During critical sessions, both objects were baited with the appropriate food on all trials. The monkeys were allowed to choose one of the objects in each pair and to obtain the reward. Two of the four critical test sessions were preceded by a selective satiation procedure, described below, one for each food. The other two were preceded by no satiation procedure and provided baseline measures of the monkeys choices. At least $2 \mathrm{~d}$ of rest followed each session that had been preceded by the selective satiation procedure. In addition, between critical sessions, the monkeys were given one regular training session with the original set of 60 object discrimination problems presented for choice in the same manner as during original learning. Critical sessions occurred in the following order for each monkey: baseline; preceded by satiation with food 1; baseline; preceded by satiation with food 2 . The effect of reinforcer devaluation was quantified as a "difference score," which was the change in choices of object type (i.e., food 1 and food 2 objects) in the sessions preceded by selective satiation relative to the baseline sessions.

For the selective satiation procedure, a food box measuring $8 \times 10 \times$ $7.5 \mathrm{~cm}$ and attached to the monkey's home cage was filled with either food 1 or food 2 while the monkey was in its home cage. This took place $\sim 24 \mathrm{hr}$ after the last feeding. The monkey was allowed to eat the food without being directly observed for 15-30 min. Then the experimenter entered the room and checked the amount of food eaten. If the monkey had eaten most of the food, the food box was refilled. Whether additional food was given or not, the experimenter started observing the monkey through a window from outside the animal housing room until the monkey refrained from taking food from the food box for $5 \mathrm{~min}$. (If the monkey emptied the food box again, the box was refilled and observation continued, until the monkey refrained from eating for $5 \mathrm{~min}$.) The test session was then initiated within $10 \mathrm{~min}$. For the baseline condition, the monkey was simply taken directly from its home cage to the test apparatus without undergoing a selective satiation procedure.

Postdisconnection retest. After the second surgery (or a period of rest), the monkeys were retrained to criterion on the original set of object discriminations to the same criterion as before. Then the reinforcer devaluation tests were repeated in exactly the same manner described above (test 2).

Progressive ratio testing. An effect of the surgical disconnection of amygdala and orbital prefrontal cortex on reinforcer devaluation could result from reduced motivation or an altered ability to respond to the devalued reinforcers themselves. Accordingly, after completion of rein- forcer devaluation testing, we tested both groups of monkeys on a progressive ratio (PR) task, which provides a measure of the monkeys' willingness to work under conditions in which increasingly greater numbers of responses are required to obtain food rewards. The prediction was that all monkeys, both operated and control alike, would show normal levels of motivation, as measured by their performance on the progressive ratio task, and intact effects of satiation when directly confronted with the outcome (i.e., the sated food). That is, the monkeys in the disconnection group were expected to be able to associate objects with the value of a reinforcer when the two items (stimulus object and devalued reinforcer) were presented together repeatedly.

Test methods were adapted from Weed et al. (1999). The test apparatus was similar in size to the WGTA used for reinforcer devaluation testing, but contained a 19 inch touch-sensitive monitor screen on which visual stimuli could be presented under computer control. After the monkeys had been shaped to touch the monitor screen, formal testing began. The monkey sat in front of the monitor screen. Touches were registered, and food pellet rewards (190 mg banana pellets) for correct responses were delivered by a computer. The stimulus was a colored 45 mm typographic character, presented in the center of the screen. Initial training involved several $10 \mathrm{~min}$ sessions of fixed ratio responding. The PR schedule was then implemented. The initial response requirement was one touch of the stimulus. The response requirement progressively increased after each reinforcer delivery (two food pellets) by an incremental value that began at one and doubled after eight response requirements had been successfully completed. Sessions were a maximum of 10 min long but were automatically terminated if 2 min elapsed without a response. The PR schedule was first administered for a total of $20 \mathrm{~d}$ at the rate of $5 \mathrm{~d}$ per week to establish stable responding. Sessions in which monkeys emitted no responses were not considered in the analysis. Five additional sessions were then given to measure the effect of satiation on progressive ratio performance. Two days of baseline testing were administered before the satiation test day and, after at least $1 \mathrm{~d}$ of rest, two more after. On the satiation test day, monkeys were allowed to eat their fill of the reinforcer (banana pellets) before being taken to the test apparatus; the satiation procedure was identical to that used during the reinforcer devaluation experiment.

\section{Histology}

At the completion of the experiment, the monkeys were anesthetized with ketamine, given a lethal dose of sodium pentobarbital $(100 \mathrm{mg} / \mathrm{kg}$, i.p.), and transcardially perfused with $0.9 \%$ saline followed by a solution of $10 \%$ formaldehyde in normal saline. The brains were removed from the cranium, photographed, and cryoprotected in a solution of $20 \%$ glycerol and $10 \%$ formalin. Tissue was sectioned in the coronal plane at $50 \mu \mathrm{m}$ on a freezing microtome. Every fifth section was mounted on gelatin-coated slides, defatted, stained with thionin, and coverslipped. The lesions were plotted onto standard drawings of brain sections. The amount of damage to the cell bodies of the amygdala and to the orbital prefrontal cortex (measured in terms of volume of the structure that was damaged) was measured with the aid of a digitizing tablet (Wacom, Vancouver, WA).

The intended lesion is illustrated in Figure 2. The volume of orbital prefrontal cortex and amygdala removed in each of the four cases is given in Table 1. Plots of the lesions in each case are presented in Figure 2; photomicrographs of sections through the orbital prefrontal cortex and amygdala in case Op3 are presented in Figure 3. Although the amygdala lesions were smaller than intended in three of the four operated subjects, each of those lesions affected a large extent of the basal nucleus, the part of the amygdala that provides much of the input to the prefrontal cortex (Amaral et al., 1992; Carmichael and Price, 1995). The extent of damage to the orbital prefrontal cortex was likewise somewhat less than intended in some subjects. The section of the anterior commissure and corpus callosum was complete in all four cases. Damage to structures outside the amygdala, orbital prefrontal cortex, and forebrain commissures was minor. Such inadvertent damage was limited to the rostral $2 \mathrm{~mm}$ of the right hippocampus in case Op4 and to the rostral $2 \mathrm{~mm}$ of the dorsal portion of the right hippocampus, together with the adjacent tail of caudateventral putamen at the same anteroposterior level, in case Op3. The relationship between the extent of the amygdala and orbital prefrontal cortex removal and the behavioral findings are discussed in Results. 
Figure 2. Intended lesion and plots of the orbital prefrontal cortex and amygdala lesions in the four operated cases (Op1-Op4), shown on ventral surface views (top) and coronal sections (bottom) from a standard rhesus monkey brain. The intended lesion is shown in the leftmost column. The thick black line and small rectangle between the hemispheres in this and other ventral views indicates the extent of the section of the corpus callosum and anterior commissure, respectively; positions of the stereotaxic levels illustrated in the coronal sections are also indicated. The ventral views for Op1-Op4 show reconstructions of the extent of the orbital prefrontal cortex lesions, and are reversed to aid in matching to the individual sections (i.e., the left hemisphere is on the left). The numbers to the left of the coronal sections indicate the distance in millimeters from the interaural plane. Compare and contrast with Figure 3. Figure 2 continues.

\section{RESULTS}

\section{Discrimination learning}

The two groups of monkeys did not differ in their rate of initial acquisition of the 60 discrimination problems (mean errors to criterion: controls, 172; disconnection, 230.5; Mann-Whitney $U=4 ; p>0.05$ ) or in reacquisition of the problems after the second surgery (or rest) (mean errors to reattain criterion: controls, 0; disconnection, 15.5; Mann-Whitney $U=4 ; p>0.05$ ).

\section{Reinforcer devaluation}

In both the tests, intact control monkeys tended to avoid choosing objects overlying the satiated, presumably devalued, reinforcer in favor of the remaining objects, reflecting control over response selection by the current value of the reinforcer. As predicted, in the first test, performance of monkeys with unilateral lesions was indistinguishable from controls. Both groups of monkeys showed
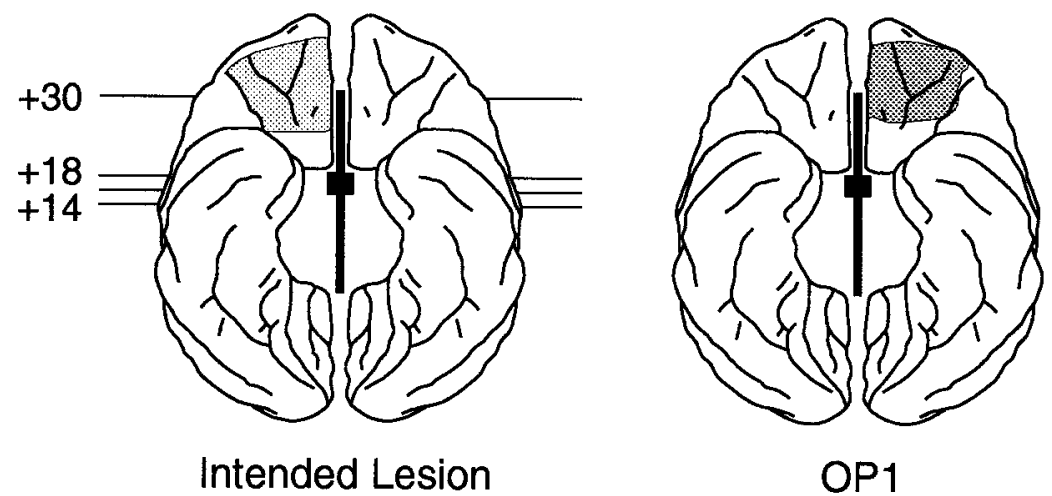

Intended Lesion
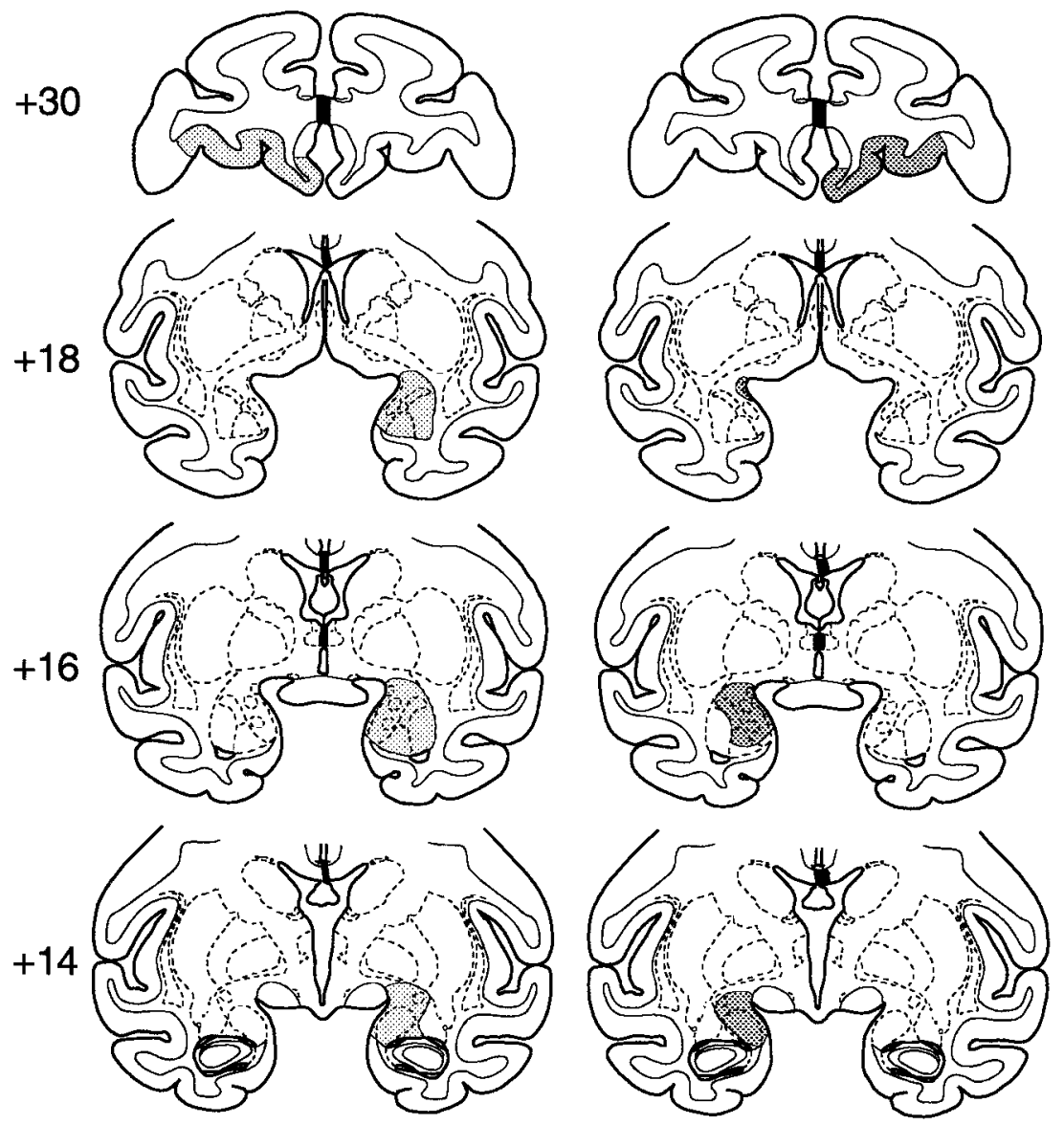

difference scores that were reliably higher than expected by chance $\left(t_{(3)}\right.$ values $>3.152 ; p$ values $<0.025$, one-tailed $)$. In the second test, after completion of the amygdala-orbital prefrontal disconnection, this effect was markedly attenuated in the operated monkeys (Fig. 4, Table 2). Indeed, every control achieved a larger difference score on test 2 relative to test 1 , whereas every operated monkey attained either the same or a lower score. Hence, monkeys with a surgical disconnection of the amygdala and orbital prefrontal cortex were unable to alter their choice behavior (i.e., selection of an object covering a particular food) in response to a change in the incentive value of the associated food.

The difference scores were analyzed using a $2 \times 2$ ANOVA with repeated measures on the disconnection stage factor, which showed no effect of group $\left(F_{(1,6)}=3.28 ; p>0.05\right)$ and no effect of predisconnection and postdisconnection stage $\left(F_{(1,6)}=0.031\right.$; $p>0.05)$. There was a significant interaction, however, between 

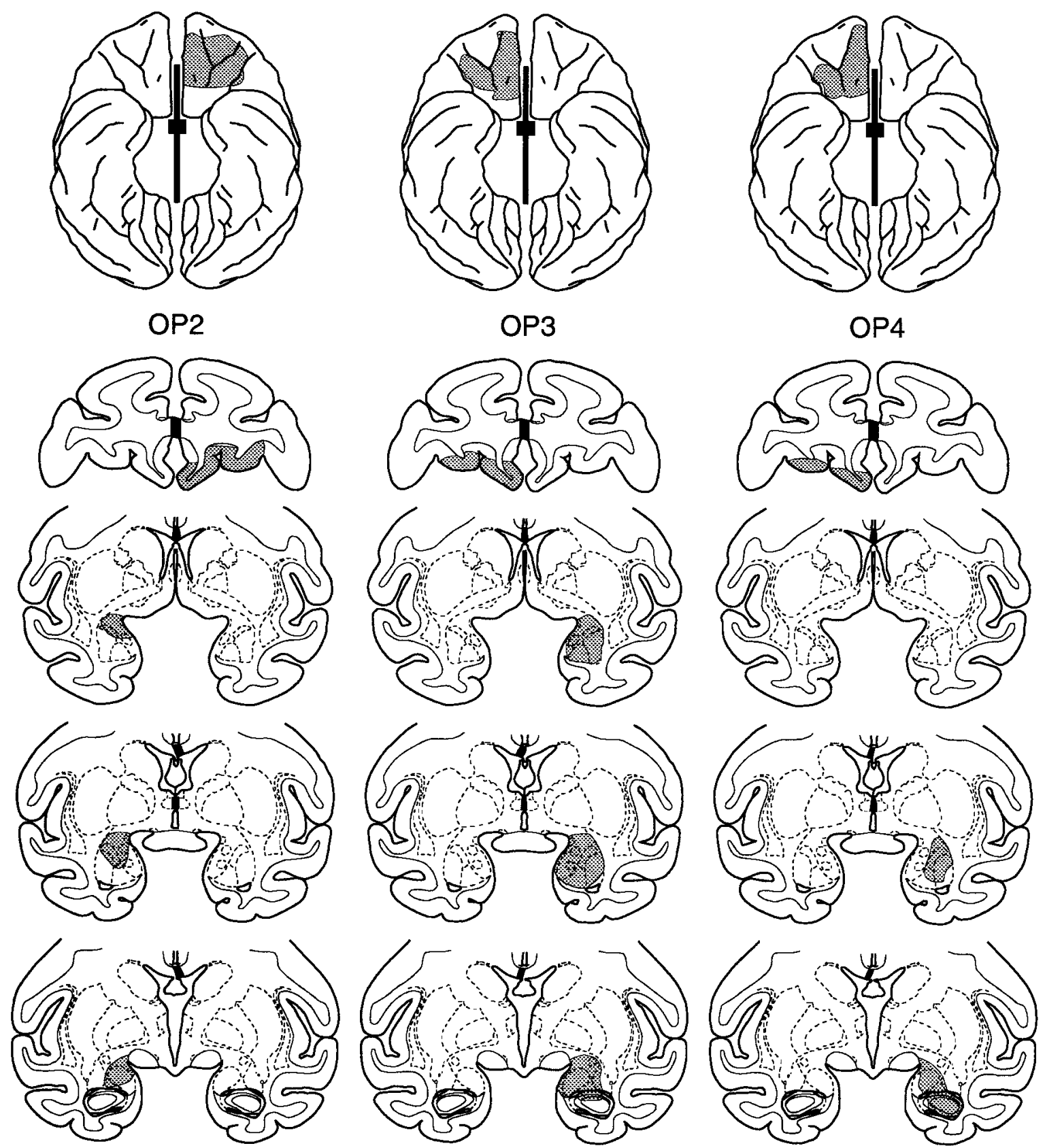

Figure 2 continued.

group and stage $\left(F_{(1,6)}=12.57 ; p=0.02\right)$, indicating that, after surgical disconnection of the amygdala and orbital prefrontal cortex, the operated monkeys chose significantly more objects covering the sated (temporarily lower-valued) food than did the controls. The better performance of the controls on test 2 relative to test 1 may be attributable to the experience gained in test 1 , which might be expected to promote learning of the specific object-food associations.

We note that there was a significant positive correlation between the extent of total amygdala damage and the decrease in scores between test 1 and test 2 in the disconnection group $\left(r_{\mathrm{s}}=\right.$ $1.0 ; p=0.03$, one-tailed); no such correlation was present between orbital prefrontal damage and impairment produced by the disconnection $\left(r_{\mathrm{s}}=0.2\right)$.

\section{Selective satiation}

Monkeys ate an average of $122 \mathrm{gm}$ of food and spent an average of $34 \mathrm{~min}$ in the devaluation procedure. Neither the amounts eaten nor the time spent in the selective satiation procedure differed between groups ( $t$ values $<1.15$; $p$ values $>0.05)$.

\section{PR testing}

Levels of motivation, measured by the maximum number of responses emitted in the PR schedule during the 20 sessions given to establish levels of responding on the standard PR task, did not differ between control and operated monkeys (controls, mean of 16.7 responses; operated, mean of 26.2 responses; $t_{(6)}=1.22 ; p=$ $0.27)$. The effect of satiation on PR performance is presented in Figure 5. Data shown are the mean number of responses emitted 
Table 1. Percent damage to amygdala and orbital prefrontal cortex in the four operated subjects

\begin{tabular}{llllll} 
& \multicolumn{2}{l}{ Amygdala } & & Orbital prefrontal \\
\cline { 3 - 3 } \cline { 5 - 6 } Case & Hemisphere & $\begin{array}{c}\text { Percent } \\
\text { damage }\end{array}$ & & Hemisphere & $\begin{array}{l}\text { Percent } \\
\text { damage }\end{array}$ \\
\hline Op1 & L (first) & 42.9 & & R (second) & 65.5 \\
Op2 & L (second) & 34.9 & & R (first) & 73.3 \\
Op3 & R (first) & 95.3 & & L (second) & 57.1 \\
Op4 & R (second) & 28.4 & & L (first) & 41.1 \\
Mean & & $\mathbf{5 0 . 4}$ & & $\mathbf{5 9 . 3}$ \\
\hline
\end{tabular}

The hemisphere in which each lesion was placed (left, L; right, R) and the order in which the surgeries were conducted are indicated. For example, case Op1 received a neurotoxic amygdala lesion in the left hemisphere before beginning behavioral testing and a lesion of the orbital prefrontal cortex in the right hemisphere, with forebrain commissurotomy in the same surgery, to complete the disconnection. See Figure 1 for a schematic of the behavioral testing sequence.

(i.e., maximum ratio attained) during the four baseline sessions combined and for the single satiation session. Both control and operated monkeys emitted a similar number of responses under baseline and satiation conditions, and all monkeys decreased their responding in response to satiation, indicating an equivalent loss of motivation in the two groups. The scores were analyzed with a $2 \times 2$ ANOVA with repeated measures on the satiation-baseline factor, which revealed no main effect of group $\left(F_{(1,6)}=0.791 ; p>\right.$ $0.05)$ or group by satiation interaction $\left(F_{(1,6)}=1.45 ; p>0.05\right)$ but a significant main effect of satiation $\left(F_{(1,6)}=14.92 ; p=0.008\right)$.

\section{DISCUSSION}

Rhesus monkeys with surgical disconnection of the amygdala and orbital prefrontal cortex, an operation intended to interrupt communication between these two structures, were unable to adjust their choice behavior in the face of altered values of the reward outcomes. Previous work had shown a similar effect after bilateral neurotoxic lesions of the amygdala in monkeys (Málková et al., 1997). In addition, related work indicated that rats with bilateral neurotoxic lesions of either the basolateral amygdala or orbital frontal cortex tested in a Pavlovian reinforcer devaluation procedure fail to reduce their levels of conditioned responding to a conditioned stimulus paired with a devalued reinforcer (Hatfield et al., 1996; Gallagher et al., 1999). The present results extend these findings by indicating that interaction between these two structures (amygdala and orbital prefrontal cortex) is required to guide choices based on the values of outcomes.

The present findings cannot be explained by deficiencies in visual perception or learning, because there were no differences between the groups in rate of learning the visual discrimination problems, or in reacquisition of the problems after the second surgery (or rest). We can also rule out the possibility that the results could be accounted for by changes in the ability of the operated monkeys to discriminate the foods or the relative values of the foods. Both operated and control monkeys showed stable preferences for objects baited with one of the foods during baseline sessions (i.e., those not preceded by satiation). That is, although we attempted to choose foods that were approximately equally preferred based on food preference tests conducted in preliminary training, there were often reliable choices of object type (food 1 objects vs food 2 objects) made during the baseline session that presumably reflected food preferences (Table 2). Hence, the values of the reinforcers per se and the relationships between specific objects and specific reinforcers were still represented in the operated group. Consistent with this idea, monkeys with bilateral lesions of the amygdala or ventromedial prefrontal cortex show stable preferences of foods from a set of familiar foodstuffs (Aggleton and Passingham, 1981; Murray et al., 1996; J. Bachevalier, personal communication). Finally, it is unlikely that
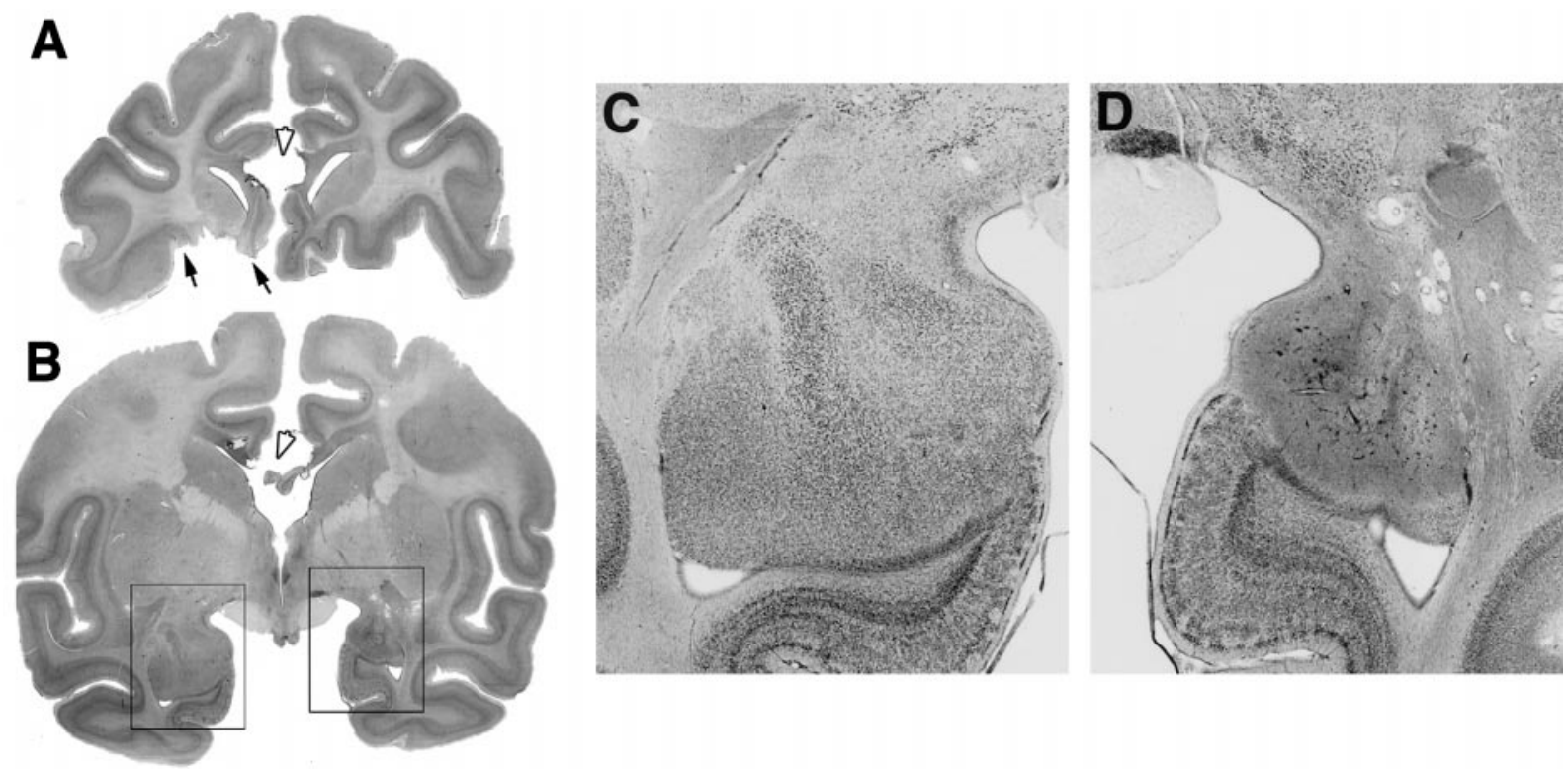

Figure 3. Photomicrographs of Nissl-stained coronal sections from case Op3. A, Coronal section $\sim 30 \mathrm{~mm}$ rostral to the interaural plane. The extent of the orbital prefrontal cortex lesion in the left hemisphere is marked by the black arrows. The section of the corpus callosum is also apparent at this level and is marked by the white arrowhead. $B$, Coronal section $16 \mathrm{~mm}$ rostral to the interaural plane. Note the marked loss in volume of the amygdala in the right compared with the left hemisphere. The section of the corpus callosum is marked by the white arrowhead. The rectangles over the left and right temporal lobes show the approximate locations of the regions shown at higher power in $C$ and $D$, respectively. $C$, Photomicrograph of the intact (left) amygdala. $D$, Photomicrograph of the right amygdala $\sim 9$ months after injection of ibotenic acid, at the same magnification used in $C$. The marked neuronal cell loss and gliosis are characteristic of excitotoxic lesions. 
Test 1

Test 2

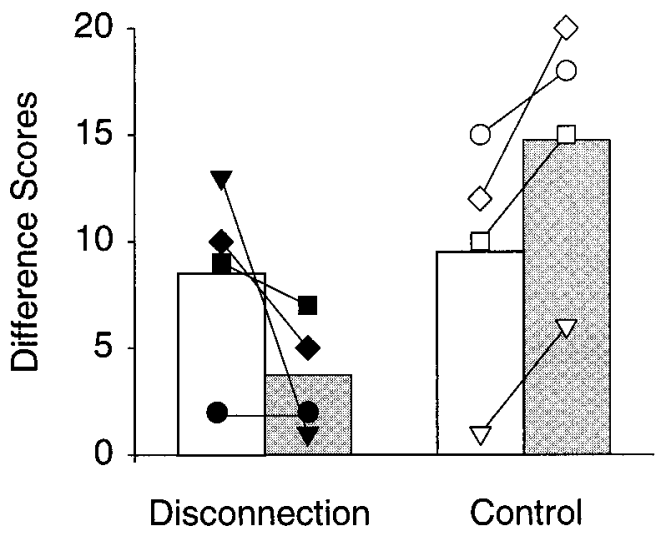

Figure 4. The effects of reinforcer devaluation performed before (Test 1) and after (Test 2) the surgical disconnection had been completed. Difference scores (devaluation - baseline) for the control and disconnection group means are shown as bars, with the symbols representing scores of individual monkeys. Normal monkeys showed an enhancement of the devaluation effect in the second test, whereas operated monkeys showed a significant reduction of this effect in the second test. Op1, Filled diamonds; Op2, filled squares; Op3, filled inverted triangles; Op4, filled circles; Con1, open diamonds; Con2, open squares; Con3, open inverted triangles; Con4, open circle.

our results can be explained by global changes in motivational levels in the operated monkeys for two reasons. First, bilateral aspiration lesions of the amygdala do not disrupt performance on the progressive ratio task (Aggleton and Passingham, 1982). Second, and more importantly, control and operated monkeys in the present study emitted a similar number of responses to obtain food on a progressive ratio schedule, a result suggesting equivalent levels of motivation in the two groups.

An important feature of the task design, one not so far remarked on, is that during each critical test session, each object was encountered only once. This means that the choice behavior toward an individual object must be based on the association in memory of a particular reinforcer with each object rather than the direct association between that object and the now devalued reinforcer encountered during that test session. As already explained, the operated monkeys were deficient, relative to controls, in making choices under these conditions. In contrast, the levels of responding in the two groups were equivalently reduced in response to a satiation manipulation, implemented in an identical manner as in the first experiment, that was superimposed on the progressive ratio task. In the latter task, unlike the former, the stimulus can be directly associated with the devalued food, because the two are presented together repeatedly. We can therefore conclude that the interaction between orbital prefrontal cortex and amygdala is critical for the control of response selection by the incentive value of the reinforcer and that the specific role of amygdala-prefrontal cortex circuitry is in associating together actions and/or objects with the representation of incentive value. Thus, the data suggest that the functional interaction of the amygdala and orbital prefrontal cortex helps animals to avoid making choices associated with adverse outcomes, without their first having to experience those adverse outcomes. If so, the neural circuitry we are uncovering may have immense biological significance.

These results, together with other experimental studies in nonhuman primates, may help explain some of the behaviors observed in humans after damage to ventral prefrontal cortex (including the orbital prefrontal cortex) or the amygdala. As mentioned at the outset, humans with damage to either of these areas are impaired in using information about the likely consequences of their actions to guide their behavior. This is true not only in their personal lives in which they often make disastrous social and financial decisions, but also as indexed by performance in a laboratory-based "gambling task" (Bechara et al., 1998, 1999; Rogers et al., 1999a). Furthermore, these same regions have been found to be activated in functional imaging studies in which humans were engaged in a gambling task (Rogers et al., 1999b). The present finding, that interaction between these areas is required for decision-making based on expected outcomes, could explain the similarity in impairments in response selection after damage to these areas in humans. Alterations in social and emotional behavior have been reported in humans with damage to either amygdala or ventral prefrontal cortex (Rolls et al., 1994; Adolphs et al., 1998; Anderson et al., 1999), changes that may be related to their cognitive impairments (Rolls et al., 1994). It is interesting to note that monkeys with neurotoxic lesions of the

Table 2. Results of reinforcer devaluation testing

\begin{tabular}{|c|c|c|c|c|c|c|c|c|c|}
\hline \multirow[b]{2}{*}{ Case } & \multicolumn{4}{|l|}{ Test 1} & \multicolumn{4}{|l|}{ Test 2} & \multirow[b]{2}{*}{$\begin{array}{l}\text { Test 2-test } 1 \\
\text { difference }\end{array}$} \\
\hline & $\begin{array}{l}\text { Baseline } \\
\text { F1:F2 }\end{array}$ & $\begin{array}{l}\text { Satiation F1 } \\
\text { F1:F2 }\end{array}$ & $\begin{array}{l}\text { Satiation F2 } \\
\text { F1:F2 }\end{array}$ & $\begin{array}{l}\text { Difference } \\
\text { score (sum) }\end{array}$ & $\begin{array}{l}\text { Baseline } \\
\text { F1:F2 }\end{array}$ & $\begin{array}{l}\text { Satiation F1 } \\
\text { F1:F2 }\end{array}$ & $\begin{array}{l}\text { Satiation F2 } \\
\text { F1:F2 }\end{array}$ & $\begin{array}{l}\text { Difference } \\
\text { score (sum) }\end{array}$ & \\
\hline Con1 & $22: 8$ & $14: 16$ & $26: 4$ & 12 & $23.5: 6.5$ & $7: 23$ & $27: 3$ & 20 & 8 \\
\hline Con 2 & $14: 16$ & $12: 18$ & $22: 8$ & 10 & $19.5: 10.5$ & $13: 17$ & $28: 2$ & 15 & 5 \\
\hline Con 3 & $27.5: 2.5$ & $28: 2$ & $29: 1$ & 1 & $20: 10$ & $13: 17$ & 19:11 & 6 & 5 \\
\hline Con4 & $21.5: 8.5$ & $7: 23$ & $22: 8$ & 15 & $15.5: 14.5$ & $6: 24$ & $24: 6$ & 18 & 3 \\
\hline Mean & & & & 9.5 & & & & 14.75 & 5.25 \\
\hline Op1 & $18.5: 11.5$ & $16: 14$ & $26: 4$ & 10 & $25: 5$ & $23: 7$ & $28: 2$ & 5 & -5 \\
\hline Op2 & $24.5: 5.5$ & $17: 13$ & $26: 4$ & 9 & $29.5: 0.5$ & $23: 7$ & $30: 0$ & 7 & -2 \\
\hline Op3 & 20.5:9.5 & $12: 18$ & $25: 5$ & 13 & $23.5: 6.5$ & $21: 9$ & $22: 8$ & 1 & -12 \\
\hline Op4 & $2.5: 27.5$ & $0: 30$ & $2: 28$ & 2 & $0: 30$ & $0: 30$ & $2: 28$ & 2 & 0 \\
\hline Mean & & & & 8.5 & & & & 3.75 & -4.75 \\
\hline
\end{tabular}

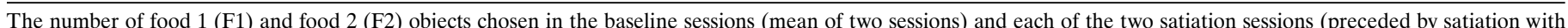

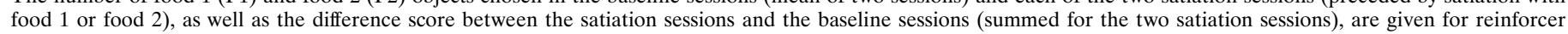
devaluation test 1 and test 2 for each of the eight monkeys (Con1-Con4, control monkeys; Op1-Op4, operated monkeys). 


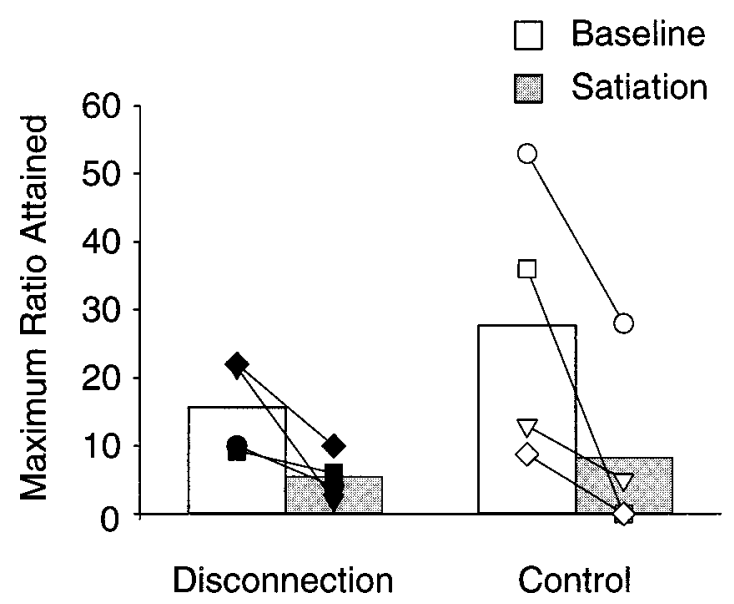

Figure 5. Results of the devaluation procedure (selective satiation) on progressive ratio testing. Baseline data shown are the mean, for each subject, of the maximum number of responses emitted (i.e., maximum ratio attained) during all four baseline sessions. Satiation data are scores for the single satiation session. Group means are shown as bars, with the symbols representing scores of individual monkeys. All monkeys, regardless of group membership, decreased responding during the test session in which the reinforcer was devalued by selective satiation. Symbols as in Figure 4.

amygdala display abnormal "emotionality," characterized primarily by inappropriate responses to evocative stimuli, including social stimuli, rather than a deficit in expressing a particular type of emotion per se (Meunier et al., 1999). It is tempting to speculate that the inappropriate emotional reactions observed after amygdala damage may represent, as in the present study, a disruption of the amygdala-orbital prefrontal system responsible for decision-making and response selection, a possibility that invites direct experimental study.

The present results do not allow us to distinguish between the different roles that the amygdala and prefrontal cortex might play in controlling response selection on the basis of reinforcer value. The amygdala is thought to play a central role in associating sensory cues with their motivational and emotional significance (Weiskrantz, 1956; Hiroi and White, 1991; Davis, 1992; Everitt and Robbins, 1992; LeDoux, 1995) and to mediate other types of associative processes as well (Holland and Gallagher, 1999). Accordingly, perhaps the deficit in the operated monkeys is attributable to a failure in the ability of the object to evoke a representation of the current value of the reinforcer. That is, the outcome associated with a particular object may be fixed in the absence of amygdala-prefrontal interaction, so the monkey does not alter its responses based on an alteration in the value of the reinforcer. Alternatively, the object may evoke the current value of the reinforcer, but this information is unable to change response selection. It is tempting to speculate that the outcome associated with a particular object is intact in the hemisphere with the intact amygdala, but this information is unable to gain control over the monkey's response selection, similar to patients with prefrontal cortex damage who report that they know they are making errors but are unable to alter their behavior accordingly (Teuber, 1972; Rolls et al., 1994). In any event, these results are consistent with models of amygdala-frontal interaction in which motivational significance, coded by the amygdala, is conveyed to the orbital prefrontal cortex for the control of action (Schoenbaum et al., 1999), as well as models of prefrontal function positing the association of objects, actions, and outcomes (Pass- ingham, 1993; Passingham et al., 2000) and for a role for the prefrontal cortex in rejecting behavior-guiding rules when those rules become maladaptive (Wise et al., 1996).

\section{REFERENCES}

Adolphs R, Tranel D, Damasio AR (1998) The human amygdala in social judgment. Nature 393:470-474.

Aggleton JP, Passingham RE (1981) Syndrome produced by lesions of the amygdala in monkeys (Macaca mulatta). J Comp Physiol Psychol 95:961-977.

Aggleton JP, Passingham RE (1982) An assessment of the reinforcing properties of foods after amygdaloid lesions in rhesus monkeys. J Comp Physiol Psychol 96:71-77.

Amaral DG, Price JL (1984) Amygdalo-cortical projections in the monkey (Macaca fascicularis). J Comp Neurol 230:465-496.

Amaral DG, Price JL, Pitkänen A, Carmichael ST (1992) Anatomical organization of the primate amygdaloid complex. In: The amygdala: neuropsychological aspects of emotion, memory, and mental dysfunction (Aggleton JP, ed), pp. 1-66. New York: Wiley-Liss.

Anderson SW, Bechara A, Damasio H, Tranel D, Damasio AR (1999) Impairment of social and moral behavior related to early damage in human prefrontal cortex. Nat Neurosci 2:1032-1037.

Bechara A, Damasio H, Tranel D, Anderson SW (1998) Dissociation of working memory from decision making within the human prefrontal cortex. J Neurosci 18:428-437.

Bechara A, Damasio H, Damasio AR, Lee GP (1999) Different contributions of the human amygdala and ventromedial prefrontal cortex to decision-making. J Neurosci 19:5473-5481.

Carmichael ST, Price JL (1995) Limbic connections of the orbital and medial prefrontal cortex in macaque monkeys. J Comp Neurol 363:615-641.

Davis M (1992) The role of the amygdala in conditioned fear. In: The amygdala: neurobiological aspects of emotion, memory, and mental dysfunction (Aggleton JP, ed), pp. 255-306. New York: Wiley-Liss.

Ettlinger G (1959) Visual discrimination following successive temporal ablations in monkeys. Brain 82:232-250.

Everitt BJ, Robbins TW (1992) Amygdala-ventral striatal interactions and reward-related processes. In: The amygdala: neurobiological aspects of emotion, memory, and mental dysfunction (Aggleton JP, ed), pp 401-429. New York: Wiley-Liss.

Gallagher M, McMahan RW, Schoenbaum G (1999) Orbitofrontal cortex and representation of incentive value in associative learning. J Neurosci 19:6610-6614.

Hatfield T, Han J-S, Conley M, Gallagher M, Holland P (1996) Neurotoxic lesions of basolateral, but not central, amygdala interfere with Pavlovian second-order conditioning and reinforcer devaluation effects. J Neurosci 16:5256-5265.

Hiroi N, White NM (1991) The lateral nucleus of the amygdala mediates expression of the amphetamine-produced conditioned place preference. J Neurosci 11:2107-2116.

Holland PC, Gallagher M (1999) Amygdala circuitry in attentional and representational processes. Trends Cognit Sci 3:65-73.

LeDoux JE (1995) Emotion: clues from the brain. Annu Rev Psychol 46:209-235.

Málková L, Gaffan D, Murray EA (1997) Excitotoxic lesions of the amygdala fail to produce impairments in visual learning for auditory secondary reinforcement but interfere with reinforcer devaluation effects in rhesus monkeys. J Neurosci 17:6011-6020.

Meunier M, Bachevalier J, Murray EA, Málková L, Mishkin M (1999) Effects of aspiration versus neurotoxic lesions of the amygdala on emotional responses in monkeys. Eur J Neurosci 11:4403-4418.

Murray EA, Gaffan EA, Flint Jr RW (1996) Anterior rhinal cortex and amygdala: dissociation of their contributions to memory and food preference in rhesus monkeys. Behav Neurosci 110:30-42.

Parker A, Baxter MG, Lindner CCC, Izquierdo AD, Murray EA (1999) Interaction of the amygdala with orbital prefrontal cortex in reinforcer devaluation in rhesus monkeys. Soc Neurosci Abstr 25:790.

Passingham RE (1993) The frontal lobes and voluntary action. Oxford: Oxford UP.

Passingham RE, Toni I, Rushworth MFS (2000) Specialization within the prefrontal cortex: the ventral prefrontal cortex and associative learning. Exp Brain Res, in press.

Porrino LJ, Crane AM, Goldman-Rakic PS (1981) Direct and indirect 
pathways from the amygdala to the frontal lobe in rhesus monkeys. J Comp Neurol 198:121-136.

Rogers RD, Everitt BJ, Baldacchino A, Blackshaw AJ, Swainson R, Wynne K, Baker NB, Hunter J, Carthy T, Booker E, London M, Deakin JF, Sahakian BJ, Robbins TW (1999a) Dissociable deficits in the decision-making cognition of chronic amphetamine abusers, opiate abusers, patients with focal damage to prefrontal cortex, and tryptophan-depleted normal volunteers: evidence for monoaminergic mechanisms. Neuropsychopharmacol 20:322-339.

Rogers RD, Owen AM, Middleton HC, Williams EJ, Pickard JD, Sahakian BJ, Robbins TW (1999b) Choosing between small, likely rewards and large, unlikely rewards activates inferior and orbital prefrontal cortex. J Neurosci 19:9029-9038.

Rolls ET (1996) The orbitofrontal cortex. Phil Trans R Soc Lond B Biol Sci 351:1433-1443.

Rolls ET (1999) The brain and emotion. Oxford: Oxford UP.

Rolls ET, Hornak J, Wade D, McGrath J (1994) Emotion-related learning in patients with social and emotional changes associated with frontal lobe damage. J Neurol Neurosurg Psychiatry 57:1518-1524.

Schoenbaum G, Chiba AA, Gallagher M (1998) Orbitofrontal cortex and basolateral amygdala encode expected outcomes during learning. Nat Neurosci 1:155-159.

Schoenbaum G, Chiba AA, Gallagher M (1999) Neural encoding in orbitofrontal cortex and basolateral amygdala during olfactory discrimination learning. J Neurosci 19:1876-1884.

Teuber H-L (1972) Unity and diversity of frontal lobe functions. Acta Neurobiol Exp 32:615-656.

Thornton JA, Malkova L, Murray EA (1998) Rhinal cortex ablations fail to disrupt reinforcer devaluation effects in rhesus monkeys (Macaca mulatta). Behav Neurosci 112:1020-1025.

Tremblay L, Schultz W (1999) Relative reward preference in primate orbitofrontal cortex. Nature 398:704-708.

Weed MR, Taffe MA, Polis I, Roberts AC, Robbins TW, Koob GF, Bloom FE, Gold LH (1999) Performance norms for a rhesus monkey neuropsychological testing battery: acquisition and long-term performance. Cognit Brain Res 8:185-201.

Weiskrantz L (1956) Behavioral changes associated with ablation of the amygdaloid complex in monkeys. J Comp Physiol Psychol 49:381-391.

Wise SP, Murray EA, Gerfen CR (1996) The frontal cortex-basal ganglia system in primates. Crit Rev Neurobiol 10:317-356. 